\section{Migrationsspezifische Aspekte in einem psychotherapeutischen Prozess}

R. Weiss

Anhand der Geschichte einer psychoanalytisch orientierten Psychotherapie mit einem lateinamerikanischen Patienten werden Themen und Konflikte erörtert, welche im Migrationsprozess gehäuft auftreten. Vor diesem Hintergrund werden praktische Hinweise für die therapeutische Arbeit mit ausländischen Patientinnen und Patienten gemacht. Insbesondere wird für den systematischen Einbezug migrationsspezifischer lebensgeschichtlicher Aspekte plädiert, ebenso für einen kulturell sensitiven Umgang mit Erklärungsmodellen zum Gesund- und Kranksein.

Herr A. muss 1989 aus seinem Land in Lateinamerika fliehen, weil er vom Militär desertiert. Er gelangt nach einer Odyssee durch zahlreiche Länder 1990 in die Schweiz, verliebt sich und heiratet. Die enge stabile Beziehung mit seiner Schweizer Frau erleichtert seine Integration, führt aber auch zu Anpassungskonflikten. Als ein Kinderwunsch konkreter wird, entwickelt er unkontrollierbare Gewaltphantasien und in der Folge panikartige Ängste. Er befürchtet, verrückt zu werden und sucht psychiatrische Hilfe in seiner Muttersprache.

Im therapeutischen Prozess verschwinden diese Vorstellungen bald, hingegen treten massive kindliche Gewalterfahrungen und narzisstische Kränkungserlebnisse in den Vordergrund. Herr A. wuchs in einer siebenköpfigen Familie auf, welche sich ohne den Vater durchbringen musste, hingegen unter dessen sporadischen Besuchen und Gewaltexzessen litt. Herr A. übernahm früh viel Verantwortung und entwickelte in der Familie eine mütterliche Rolle. Die ständige Entwertung durch den meist abwesenden Vater prägte ihn. In der Therapie bleiben damit verbundene Gefühle zunächst aber kaum zugänglich. Er lässt mich in dieser Zeit kaum zu Wort kommen und ich werde mir der Grenzen meiner Spanischkenntnisse immer bewusster. Einer Deutung aufgrund meiner entsprechenden Gegenübertragung mit Insuffizienzgefühlen folgt ein Traum, in welchem tiefer Hass

Korrespondenz:

Dr. med. Regula Weiss

Kanzleistrasse 53

CH-8004 Zürich

E-mail: regula.weiss@access.ch und Mordphantasien gegenüber dem Vater deutlich werden. Die therapeutische Beziehung entspannt sich und es wird möglich, ödipale und narzisstische Konflikte in gegenwärtige Belastungssituationen zu übersetzen und aufzuarbeiten. Die berufliche Situation von Herrn A. ist in dieser Zeit von entmutigenden und entwertend erlebten Schranken geprägt, er traut sich nicht, für seine Angelegenheiten offen einzustehen. Zugleich nehmen eheliche Konflikte zu und er schliesst die Möglichkeit, Vater zu werden, vorerst aus. Der Ausweg, die beruflichen Ziele in der Schweiz aufzugeben, seine Frau zu verlassen und in sein Heimatland zurückzukehren, wird zur zentralen Phantasie.

Zusätzlich belastet ihn eine chronisch-rezidivierende prostatische Schmerzstörung, welche zu unergiebigen Abklärungen führt. Ihre somatoformen Wurzeln zu klären, ist schwierig, eröffnet aber den Zugang zu Herrn A.s persönlichen und kulturspezifischen Erklärungsmodellen. Er verknüpft die Tatsache, dass er seiner damals ungeplant schwangeren Freundin vor seiner Flucht aus Vernunftgründen zu einer Abtreibung geraten hatte, mit der Möglichkeit eines bösen Zaubers, den die Verlassene gegen ihn ausgesprochen habe. Schuldgefühle sowie eine allgemeine Hilflosigkeit machen sich breit. Ich erkläre, nur wenig über entsprechende, kulturgebundene Behandlungsmethoden zu wissen und ermuntere ihn, mir diese zu erläutern. Er beschreibt diverse Heilpraktiken seiner Mutter, welche grosse Kräfte besitze, sowie von anderen Heilern, von denen er sich sporadisch behandeln lässt. Das Thema ist aber schambesetzt und wird bald wieder verlassen.

Die ehelichen Konflikte nehmen überhand und führen schliesslich zur Scheidung. Zur selben Zeit schliesst Herr A. trotz grosser Selbstzweifel und Prüfungsängste erfolgreich eine berufsbegleitende Ausbildung ab und findet problemlos eine Wunschstelle. Parallel dazu nehmen die Auseinandersetzungen mit seinen Geschwistern im Herkunftsland einen schärferen Ton an, seine Bindung an die Familie verändert sich. Er beginnt sich von der alleinigen finanziellen Verantwortung für die Familie zu distanzieren und Rückkehrphantasien gegen die realistischen Vorteile eines Verbleibs in der Schweiz abzuwägen. Dann verliebt er sich und zieht bald mit der neuen Freundin zusammen.

Im Verlauf von zwei Therapiejahren sind tiefe narzisstische Ängste und Zweifel an einer männlichen Identität einer stabileren Selbstbesetzung gewichen. Die ursprünglichen, bedrohlichen Gewaltphantasien kehren jedoch wieder, als in der neuen Beziehung erneut die Kinderfrage auftaucht. Diesmal kann er sich aber den inneren Verfolgern stellen und steigt auf mein Angebot eines Settingwechsels (Couch, Frequenzerhöhung) ein, um seinem "Monstrum» auf den Grund zu gehen. Es wird möglich, kulturgebundene Erklärungskonzepte genauer zu erörtern und entsprechende Konflikte, Ängste und Praktiken in das Netz an Bedeutungen zu integrieren, das Herr A. inzwischen aufgebaut hat. Das Reden über unsichtbare Kräfte wirkt triangulierend und mein Interesse an den am afrikanischen Hintergrund seiner Mutter orien- 
tierten Vorstellungen und Praktiken stärkt sein Selbstvertrauen. Er vermag nun seine Phantasien immer konkreter $\mathrm{zu}$ beschreiben und beginnt seine identifikatorischen Ängste mit dem gequälten Kind und die abgewehrten Mordgelüste zu verstehen. Die unerträglichen Affekte können integriert und das väterliche Introjekt nun auch ohne Gewaltphantasien besänftigt werden. Herr A. beginnt in seinem Alltag, eigene synchretistische Gesundheitskonzepte und Praktiken zu verfeinern, sie gegenüber der Freundin $\mathrm{zu}$ verteidigen und ist zunehmend besser dazu in der Lage, seinen inneren und äusseren Dämonen die Stirn zu bieten. Die im Zusammenhang mit sozialen Konflikten auftretenden Ängste können nun gemildert und Ich-dyston erlebte, aggressive Affekte integriert werden.

Welche Aspekte dieser interkulturellen Psychotherapie verweisen auf den Migrationsprozess? Bei Herrn A. zeigen sich zunächst universale Konflikte, die ich nicht als migrationsspezifisch bezeichnen würde, wenn sie auch aufgrund typischer Alltagserfahrungen von Migrantinnen und Migranten vermutlich bevorzugt reaktiviert werden: Es geht um das Verhältnis von Macht und Ohnmacht, von Täter und Opfer, von Abhängigkeit und Autonomie, von Altruismus und Egoismus, von Mann und Frau. Es entwickelt sich eine individuell geprägte Übertragungsbeziehung, in der ich sowohl Anteile des bedrohlich-invasiven Vaters wie auch der omnipotenten Mutter annehme.

Daneben werden aber Themen deutlich, die tatsächlich typisch sind für Menschen im Migrationsprozess. In der Migration gehen nicht nur zentrale soziale Beziehungen verloren, auch vorher geteiltes Alltagswissen büsst seine Funktion ein. Der lange Übergangsprozess geht mit einer langdauernden psychischen und sozialen Labilisierung einher. Eine der Überlebensstrategien von Migrantinnen und Migranten besteht darin, sich einen psychischen Schonraum einzurichten, um ihre Identität zu erhalten und in der neuen Lebenswelt zu entwickeln. Diese Zwischenwelt [1] schafft Voraussetzungen für den Prozess der Ablösung und für eine soziale Neuorientierung. In ihr werden in kreativer Weise alte soziale Konzepte neuen gegenübergestellt, welche sich konkurrenzieren, sich gegenseitig durchdringen und nebeneinander koexistieren.

Typisch ist auch die Mobilisierung von narzisstischen Selbstbesetzungen, ähnlich denjenigen während der Adoleszenz. Man muss sich etwas intensiv wünschen und auch zutrauen, um den Schritt in die Migration zu tun und um mit ihren Verunsicherungen kreativ umzugehen. Diese narzisstische Energie macht Veränderungen möglich, erhöht aber auch die Gefahr von Kränkungen. Kulturell, individuell und geschlechtsspezifisch geprägte Vorstellungen bestimmen, welche sozialen und ökonomischen Ziele besonders erstrebenswert sind. Für Migrantinnen und Migranten erweist sich die Kluft zwischen eigenen Zielerwartungen und dem real erreichbaren Status oft als besonders tief. Der schlechte Zugang zu gesellschaftlichen Ressourcen gelangt immer wieder ins Bewusstsein und gefährdet das narzisstische Gleich- gewicht. Verschärfend wirken sich Erwartungen von Familienangehörigen im Herkunftsland aus, welche oft unrealistische Vorstellungen über die ökonomischen Möglichkeiten von Migrantinnen und Migranten in der Schweiz haben. Im Falle von patriarchalen familiären Rollenstrukturen stehen Männer und Jugendliche dabei unter einem besonderen Druck.

Narzisstische Konflikte können sich in Form depressiver oder aggressiver Symptome äussern, als Scham oder in einer Verschiebung auf somatische Beschwerden. Sie sind in Psychotherapien mit Migrantinnen und Migranten häufig. Die Präsentation körpernaher Symptome wird dabei von Behandelnden oft als kulturspezifisches Problem verstanden. Dabei ist die Gefahr gross, das Fremde im ausländischen Patienten zu exotisieren, seine Konflikte zu kulturalisieren und demgegenüber den Einfluss realer sozialer Bedingungen zu unterschätzen, in denen Migrantinnen und Migranten leben. Hier sind schichtspezifische Belastungsfaktoren (schwere Arbeit, gehäufte Unfälle, beengte Wohnverhältnisse, doppel- und dreifachbelastete Frauen) meist aufschlussreicher als herkunftsspezifische Überlegungen. Letztere bleiben allerdings für die Art der Symptompräsentation sowie für das Gesundheits- und Krankheitsverhalten von Bedeutung. Stress wird in den meisten Gesellschaften somatisiert, besonders in südlichen und nicht westlichen Ländern sowie in Situationen der psychosozialen Entwurzelung, wenn familiäre oder vertraute Hilfssysteme nicht genügen. Die Medikalisierung der psychosozialen Misere von Migrantinnen und Migranten repräsentiert die allgemeine Tendenz, soziale Konflikte an das Gesundheitswesen zu delegieren. Das trifft übrigens für alle marginalisierten Gruppen $\mathrm{zu}$, nicht nur für Migrantinnen und $\mathrm{Mi}-$ granten.

Welche Symptome vorherrschen und präsentiert werden, ist sozialisations- und kontextabhängig. Somatisierungen sind mit komplexen Bedeutungssystemen verknüpft, welche diverse psychische und soziale Funktionen haben. Deshalb werden sie verschieden erlebt und präsentiert und gelten als eigentliche «idioms of distress» [2]. Je nach Umständen werden körpernahe Symptome als Ausdruck von Krankheit oder Psychopathologie verstanden, als symbolische Kondensation eines intrapsychischen Konfliktes, als kulturell kodierter Ausdruck von Leiden, als Medium zur Verdeutlichumg sozialen Unwohlseins oder als Mechanismus, mit dem Patientinnen und Patienten sich innerhalb ihrer lokalen Welt positionieren. Gewisse Gefühle werden sozial besser akzeptiert als andere. Emotionen sind Ausdruck davon, wie man sich in Beziehung zu anderen fühlen und verhalten sollte und damit in eine kulturspezifische, soziale Ideologie eingebettet [3]. "Sozial schlechte" Gefühle wie Wut, Angst, Neid, Habsucht, Ekel werden meist externalisiert und als durch äussere Faktoren hervorgerufen verstanden.

Wut und Enttäuschung angesichts erschwerter Partizipationsmöglichkeiten und nicht realisierter sozialer Aufstiegswünsche können bei Migrantinnen und Migranten ein erhebliches Ausmass annehmen. 
Eine depressive Verarbeitung führt nur selten zu hilfreichen Reaktionen der Umgebung. Demgegenüber ist eine somatoforme Verarbeitung erfolgreicher, weil damit verbundene Appelle gut akzeptiert sind und Verzweiflung verstanden wird. Ausserdem werden mit der Verschiebung von sozial schlechten Gefühlen in funktionsgestörte Organe, mit der Verdinglichung der narzisstischen Depression die inakzeptablen Gefühle vom Selbst abgetrennt [4]. Aber auch auf der Seite der Behandelnden kann mit der Diagnose einer somatoformen Störung die Verantwortung für das Kranksein, für Verschlechterungen oder fehlenden therapeutischen Erfolg auf den Patienten/die Patientin oder auf verdinglichte Teile seines/ihres Selbst übertragen werden [5]. Es muss hier unbedingt zwischen körpernaher Symptompräsentation, welche der Beziehungsaufnahme dient und etwas über Erwartungen aussagt, und somatoformen Fixierungen unterschieden werden, mit welcher sich eine als auswegslos erlebte Kränkungssituation in einer ungünstigen Verschiebung zu verfestigen beginnt. Sonst entsteht der - häufig zitierte - falsche Eindruck, Migrantinnen und Migranten würden generell zur Somatisierung neigen.

Migrantinnen und Migranten benutzen für ihr Kranksein oft Erklärungsmodelle, welche sich von denjenigen der Behandelnden deutlich unterscheiden und diesen kaum bekannt sind. Erklärungsmodelle dienen (den Patientinnen/Patienten und den Behandelnden!) dazu, dem Leiden Bedeutung zu geben, und mittels geeigneter Praktiken das Gleichgewicht zwischen inneren und äusseren Kräften zu beeinflussen, handlungsfähig zu bleiben. Es lohnt sich, diese einzubeziehen, um divergierende Erwartungshaltungen abzubauen, Konflikte zu verringern und damit Ressourcen von Patientinnen und Patienten zu mobilisieren. Die Konsultation wird dann zur Plattform, auf der gemeinsame Konzepte ausgehandelt werden, welche die Behandlung bestimmen [6]. Patientinnen und Patienten nutzen verschiedene Gesundheitsangebote, welche das formelle Medizinalsystem ergänzen. Unsere Beziehung zu ihnen bleibt immer eine unter vielen. Migrantinnen und Migranten greifen in der Not meist auch auf biomedizinische und alternative Heilmethoden im Ursprungsland zurück, welche ihnen von Angehörigen vermittelt werden. Wir tun gut daran, diese Aktivitäten nicht ausschliessen oder bestimmen zu wollen, sondern sie dabei integrativ zu begleiten. Besonders wenn weder kulturelle Hintergründe zum Kranksein noch typische soziale Netzstrukturen bekannt sind, ist es nützlich, Fragen zum sozialen Bedeutungszusammenhang zu stellen, an welchem sich die Person mit ihren Symptomen orientiert.

Die Interaktion mit ausländischen Patientinnen und Patienten wird aber auch von unseren Erklärungsmodellen sowie unserer beruflichen Identität geprägt. Die westlichen medizinischen und psychotherapeutischen Konzepte, die unser Denken strukturieren, sind kulturgebundene, soziale Konstrukte. In den Auseinandersetzungen im Behandlungszimmer manifestieren sich auch Konflikte innerhalb plurali- stischer medizinischer und psychologischer Referenzsysteme und unser persönliches Verhältnis zu ihnen. In den Konzepten und Zielen der westlichen Psychotherapie werden Werte wie Autonomie und Individualität betont und ein partnerschaftliches Arbeitsbündnis angestrebt. Diese Konstrukte prägen unsere Haltung Patientinnen und Patienten gegenüber. Migrantinnen und Migranten aus südlichen oder nichtwestlichen Gesellschaften haben aber oft ganz andere Erwartungen an uns. Häufig sollen wir die Rolle einer Autoritätsperson einnehmen, die über den therapeutischen Verlauf bestimmt, das Kranksein legitimiert und die Hauptverantwortung für den Genesungsprozess übernimmt. Oder wir werden als Figuren mit magischen Kräften wahrgenommen. Bei migrierten Angehörigen der städtischen Mittelschicht spielen kulturspezifische Unterschiede allerdings eine verhältnismässig geringe Rolle, weil sie uns sozial nahestehen.

Nicht zuletzt wiederholen und inszenieren sich Kränkungen im Migrationsprozess in unserer Interaktion mit ausländischen Patientinnen und Patienten. In den typischen, manchmal schwer zu ertragenden Gegenübertragungsreaktionen von Behandelnden spiegelt sich nicht selten die Ohnmacht, Lähmung und latente Wut aufgrund von kaum beeinflussbaren Alltagserfahrungen von Migranten. Anderseits kann ein stabiler therapeutischer Raum als Teil jener Übergangswelt genutzt werden, in der die Rekonstruktionen der Identität stattfinden. In ihm können migrationsspezifische Konflikte in der Übertragung bearbeitet werden.

Wie können nun im therapeutischen Kontakt gesundheitsrelevante Aspekte im Migrationsprozess fokussiert werden? Es kann hilfreich sein, neben der jeweiligen psychotherapeutischen Methode sich an einem Raster zu orientieren, in welchem sich Erfahrungen einordnen lassen und von welchem aus diese gezielt erfragt werden können. Dadurch kann die Sensibilität gegenüber typischen Stressoren aber auch nutzbaren Ressourcen verbessert werden. Eine migrationsspezifische Perspektive sollte sich auf drei Phasen richten, welche die Geschichte von Migrantinnen und Migranten prägen. Sie sind vor allem bei der Anamneseerhebung, aber auch für das Erkennen von Konflikten im therapeutischen Prozess von Bedeutung:

\section{Prägende Aspekte im Migrationsprozess [7, 8]}

\section{Prämigrationsgeschichte (Herkunft)}

- sozialer Kontext:

Lebenssituation (Kernfamilie, Verwandtschaft), soziales Umfeld,

schulische und berufliche Entwicklung, ökonomische Situation;

- psychosoziale Entwicklung: zentrale Objektbeziehungen, Trennungs- und Verlusterfahrungen, Traumatisierungen;

- politische Situation;

- religiöse Praxis; 
- Gesundheitsverhalten: praxisrelevante Erklärungsmodelle, vorhandene Behandlungsangebote und deren Nutzung.

\section{Migrationsgeschichte}

- Motivationen für die Migration: persönliche Wünsche und Hoffnungen, ökonomische/politische Zwänge;

- Umstände und Verlauf:

Dauer,

Stationen,

Beteiligte;

- Traumatisierungen.

\section{Postmigrationsgeschichte (Integration)}

- rechtliche Lage:

Aufenthaltsstatus,

Arbeitsbewilligung;

- psychosoziale Situation:

Arbeits- und Wohnsituation,

lokales Netzwerk,

Trennungs- und Verlusterfahrungen,

Remigrationsperspektiven,

sprachliche Integration,

Diskriminationserfahrungen

Ausgestaltung von Übergangswelten zwischen Herkunfts- und Residenzgesellschaft;

- transnationale Netzwerke:

Struktur und Stabiltät des Familiensystems, grenzüberschreitende Kontakte und Bindungen;

- ökonomische Situation:

finanzielle Möglichkeiten,

Verpflichtungen gegenüber der Ursprungsfamilie, Schulden;

- Gesundheitsverhalten:

Nutzung von Angeboten des Gesundheitssystems, praktische Bedeutung herkunftsbezogener Erklärungsmodelle.

\section{Literatur}

1 Hettlage-Varias A, Hettlage R. Kulturelle Zwischenwelten. Fremdarbeiter - eine Ethnie? Sonderheft der Schweizerischen Zeitschrift für Soziologie 1984;10:357-403.

2 Kirmayer LJ, Young A. Culture and somatization: clinical, epidemiological, and ethnographic perspectives. Psychosom Med 1998;60:420-30.

3 Myers FR. Emotions and the Self: A Theory of Personhood and Political Order among Pintupi Aborigines. Ethos 1979; 7:342-70.

4 Cassel EJ. Disease as an "It": Concepts of Disease Revealed by Patients presentation of Symptoms. Soc Sci Med 1976; 10:143-146.

5 Good BJ, Del Vecchio Good M-J. The Semantics of Medical Discourse. In: Mendelsohn E, Elkana Y (eds.). Sciences and Cultures. Dordrecht: Reidel; 1981. p. 177-212.

6 Kleinman A. Patients and Healers in the Context of Culture: An Exploration of the Borderland between Anthropology, Medicine and Psychiatry. Comparative Studies of health systems and medical care, no. 3. Berkeley, University of California Press; 1980. pp. 427.

7 Salis Gross C, Moser C, Zuppinger B, et al. Die Arzt-PatientenInteraktion aus der Sicht von MigrantInnen: Vorschläge für die ärztliche Praxis. Schweizerische Rundschau für Medizin (PRAXIS) (Sondernummer Migration und Gesundheit Teil 2) 1997;86(21):887-94.

8 Salis Gross C, Sabbioni M. Die migrationsspezifische Anamnese: Konzeptuelle und praktische Implikationen. In: Adler R, Sabbioni M (Hrsg.). Die Anamnese: Theorie und Praxis. Stuttgart: Gustav Fischer Verlag; 1999. 